\title{
A randomized control study to assess the efficacy of platelet rich plasma and local corticosteroid injection in treatment of chronic plantar fasciitis
}

\author{
K.M. Gopinath ${ }^{1}$, Bharath.$S^{2, *}$, Roshan Kumar B. ${ }^{3}$ \\ ${ }^{1}$ Professor, ${ }^{2}$ Junior Resident, ${ }^{3}$ Professor and HOD, Dept. of Orthopaedics, Rajarajeshwari Medical College and Hospital, \\ Bengaluru, Karnataka, India
}

*Corresponding Author:

Email: bharathdoc.4176@gmail.com

\begin{abstract}
Introduction and Objectives: Among all the known tendinopathies plantar fasciitis and tennis elbow account for most cases.Corticosteroid injections considered the treatment of choice for tendinopathies. Emerging therapeutic modalities is the use of platelet rich plasma for tendinopathies having the advantage of being autologous and concentrated that are rich in growth factor proteins. The study was done to find the efficacy of these two modalities.

Materials and Methods: The study was done at Rajarajeshwari Medical College and Hospital, satisfying the inclusion criteria and exclusion criteria. In this study, $3 \mathrm{ml}$ of the extracted PRP or $40 \mathrm{mg}$ of prednisolone was injected into the affected area. Postintervention pain and functional assessment at 2 weeks, 6 weeks, 3 months and 6 months with visual analogue score and ankle hind foot scale and foot and ankle disability index score.

Results: The age in our study was 18 to 55 years, the mean in the steroid group was 37.3 and in the PRP group was 37.43 , right side was most commonly involved $52 \%$. In both the groups there was significant difference in the VAS scores $\mathrm{p}<0.001$ from the time of presentation to the first follow up in both the groups this further improved at the second follow up significant difference $\mathrm{p}<0.001$ after which it remained constant, between the two groups there was no difference with a chi-squares $\mathrm{p}$ valueless than 0.05 at all times.

In both the groups there was significant difference $\mathrm{p}<0.001$ from the time of presentation to the first follow up in both the groups FADI score.

Interpretation and Conclusion: PRP has better effect pain in patients with longer duration as compared to local steroids but the difference is not statistically significant.

The making of PRP is cumbersome but the patients did prefer to steroids due to the adverse effects.
\end{abstract}

Keywords: FADI- Foot and ankle disability index score, PRP- Platelet-rich plasma.

\section{Introduction}

Plantar fasciitis is the commonest cause of foot complaints in India. The incidence of plantar fasciitis peaks in people between the ages of 40 to 60 years with no bias towards either sex. ${ }^{1}$ It is a degenerative tissue condition that occurs near the site of origin of the plantar fascia at the medial tuberosity of the calcaneous. ${ }^{2}$

Histological findings show no evidence of inflammation; rather, they show myxoid degeneration, micro tears, collagen necrosis, and angiofibroblastic hyperplasia. These findings suggest a chronic degenerative process, not an acute inflammatory one. ${ }^{3}$

Conservative treatment for plantar fasciitis in the form of stretching, nonsteroidal anti-inflammatories, night splinting, strapping, orthoses, and shoe modifications are effective for $90 \%$ of cases. Ten percent remain recalcitrant to these treatments necessitating more aggressive intervention including injection therapy, extra corporeal shock wave therapy (ESWT), and in some instances surgical release of the origin of the plantar fascia. ${ }^{4}$

Although the effect of ESWT remains controversial, reliable evidence supports the use of this approach for treating chronic plantar fasciitis, ${ }^{5,6}$ However, adverse effects such as pain during treatment, soft tissue damage (bleeding, hematoma, paresthesia), nausea, the need for peripheral nerve block and costs should be considered when proposing this procedure ${ }^{7}$.

Steroid injections are a popular method of treating the condition but are useful in the short term and only to a small degree. ${ }^{8}$ However, the lack of an inflammatory process histologically in plantar fasciitis questions its mode of action. . $^{9}$

Recently, promising results were reported with the use of platelet-rich plasma (PRP) injections for treating muscle and tendon injuries and degeneration..$^{10-16}$

The use of autologous PRP was first used in 1987 by Ferrari, et al. ${ }^{17}$ PRP is a bioactive component of whole blood with platelet concentrations elevated above baseline and containing high levels of various growth factors. ${ }^{18}$ The rationale for PRP benefit lies in reversing the blood ratio by decreasing red blood cells (RBC) to $5 \%$, which are less useful in the healing process, and increasing platelets to $94 \%$ to stimulate recovery. ${ }^{19} \mathrm{An}$ increased awareness of platelets and their role in the healing process has led to the concept of therapeutic applications. There is a emerging literature on the beneficial effects of PRP for chronic non-healing tendon injuries including lateral epicondylitis and plantar fasciitis. $^{20,21}$

PRP increases tendon regenerative abilities with a high content of cytokines and cells, in hyper physiologic doses, which should promote cellular 
chemotaxis, matrix synthesis, and proliferation. $^{22}$ Degranulation of the alpha granules in platelets releases many different growth factors that can play a role in tissue regeneration processes. PRP represents a treatment option for many foot and ankle pathologies, including tendinopathy (Achilles, peroneal, posterior tibial, flexor hallucis longus, anterior tibial) and chronic ligamentous injury, such as plantar fasciitis. ${ }^{23}$

In view of the above side we conducted a study entitled as "A randomized control study to assess the efficacy of platelet rich plasma and local corticosteroid injection in treatment of chronic plantar fasciitis" with an aim to assess the efficacy of PRP injections and local corticosteroid injection for treating chronic plantar fasciitis and provide initial clinical assessment of its effectiveness.

\section{Materials and Methods}

Place of Study: Outpatient Department of Orthopaedics, RRMC\&H

Study Subjects: Patients with chronic plantar fasciitis satisfying inclusion criteria.

Study Design: Randomized control study.

Study Period: December 2015 to November 2016.

Sample Size Estimation: Sample size 60

Informed consent has been taken from every patient and ethical committee clearance taken from institute.

Inclusion Criteria: Patients aged $\geq 18$ years, experienced heel pain felt maximally over the plantar aspect for at least three months continuously and had radiographic evidence of calcaneal spur and failed to respond to conservative management.

Patients who are willing to participate in the study.

Exclusion Criteria: Patients of plantar fasciitis in presence of other systemic disease like diabetes mellitus, rheumatoid arthritis, and gout.

Hemoglobin $<5.0$.

Physical/occupational therapies within 4 weeks.

Any wound or skin lesion at the plantar aspect of the foot.

Pregnancy

Severe infection, known malignancy, bleeding disorder, previous surgery.

Nerve-related symptoms such as radiculopathy, tarsal tunnel syndrome or tarsi sinus syndrome; foot and ankle osteoarthritis.

\section{Method of Collection of Data}

Diagnosis: On reviewing the literature it was found that the diagnosis of chronic plantar fasciitis is mostly based on history and clinical examinations and the following symptoms have been reported $:^{24}$

1. The condition typically presents with sharp morning heel pain and "first-step" pain that improves with use during the day and often worsens with heavy use.

2. Other symptoms such as numbness, tingling, night pain, swelling, and radiating pain.
3. The symptoms of plantar fasciitis often begin after exercise or activity modification, shoe change, weight gain, or minor foot injury.

\section{Radiological Findings}

X-Ray: Plain radiographs of the hind foot may confirm calcifications or spur formation in chronic cases. The clinical importance of calcaneal spurs is uncertain, but it is clear that they are not the cause of plantar fasciitis and an adaptive, physiologic response to chronic traction and irritation in the injury zone. ${ }^{24}$

Ultrasound: A more cost-effective and engaging technique for imaging plantar fasciitis is ultrasonography. Although operator-dependent, the technology allows the rapid, reproducible, and accurate diagnosis of plantar fasciitis. The technique is painless and quick. Typical findings seen on ultrasound are hypo echoic islets and disrupted fascial bundles. One particular advantage of ultrasound over other techniques is that it allows cost-effective interval studies to document the effects of clinical intervention. $^{24}$

\section{PRP Concentrate Preparation}

Autologous PRP preparation: Under strict aseptic precautions 10cc of whole blood will be drawn by venepuncture into $10 \mathrm{cc}$ syringes with $\mathrm{ACD}-\mathrm{A}$ as an anticoagulant.

Centrifugal force separates the blood components into three distinct layers based on their particular densities The heaviest particles, the red blood cells sunk at the bottom of the tube, the least dense constituents the platelet-poor plasma (PPP) move to the top of the tube, while the platelet-rich plasma (PRP) remained at the center.

The whole blood is initially centrifuged by placing the syringe directly into the Centrifuge. This first spin is called 'soft spin' at 3000rpm for 3 minutes. This causes Separation of the blood into RBC, buffy coat and platelets and top platelet poor plasma. The upper layer of plasma including the platelets and buffy coat is drawn into another syringe using a 3 way connector. This is subjected to the second centrifugation called 'hard spin' at 4500rm for 15 minutes.

This causes separation of the platelets and platelet poor plasma. From this the supernatant plasma can be discarded and the platelets can be re-suspended in appropriate volume of either plasma or normal saline.

\section{Observations and Results}

The study is a comparative case control randomized observational prospective study the data of which was collected from patients attending outpatient and inpatient services in Rajarajeshwari Medical College and Hospital, Bangalore to the Department of Orthopaedics beginning from December 2015 to November 2016 on those cases with chronic plantar 
fasciitis who have satisfied the inclusion criteria and exclusion criteria.

Groups in the Study: In our study we had a total of 60 cases, 30 in the steroid group and 30 in the PRP group.

Table 1: Age

\begin{tabular}{|l|c|c|}
\hline Age & Frequency & Percentage \\
\hline less than 20 & 2 & 3 \\
\hline $21-30$ & 7 & 12 \\
\hline $31-40$ & 32 & 53 \\
\hline $41-50$ & 16 & 27 \\
\hline $51-60$ & 3 & 5 \\
\hline $61-70$ & 0 & 0 \\
\hline total & 60 & 100 \\
\hline & Age & P Value \\
\hline PRP & 37.43 & 0.99 \\
\hline Steroid & 37.3 & Not Significant \\
\hline
\end{tabular}

The least age in our study is 18 years and the maximum age is 55 years, the mean age in the steroid group is 37.3 and in the PRP group is 37.43 , the most common group is $31-40$ years.

Table 2: Gender

\begin{tabular}{|l|c|c|c|c|}
\hline \multicolumn{2}{|l|}{ Groups } & Frequency & Percent & Total \\
\hline PRP & Female & 7 & 23.3 & Females, 16, males 44 \\
\cline { 2 - 5 } & Male & 23 & 76.7 & 60 \\
\cline { 2 - 5 } & Total & 30 & 100 & P Value \\
\hline \multirow{3}{*}{ Steroid } & $\mathrm{F}$ & 9 & 30 & 0.85 \\
\cline { 2 - 5 } & $\mathrm{M}$ & 21 & 70 & Not Significant \\
\hline
\end{tabular}

In the present study overall males appeared to be more affected with plantar fasciitis in both the groups there is statistically significant difference, hence the two groups were comparable.

Side: In the steroid group 6 were bilateral, 9 were left and 15 were right side respectively and in the PRP group 2 bilateral, 12 were left and 16 were right side respectively. Right side is most commonly involved $52 \%$.

Occupation: The most common occupation involved was (farmers 32\%) followed by (shop keepers 22\%) and then (teachers 17\%).

Table 3: VAS - AT various intervals

\begin{tabular}{|l|c|c|c|c|c|}
\hline & $\begin{array}{c}\text { VAS -At } \\
\text { presentation }\end{array}$ & $\begin{array}{c}\text { VAS - 1st } \\
\text { follow up }\end{array}$ & $\begin{array}{c}\text { VAS - 2nd } \\
\text { follow up }\end{array}$ & $\begin{array}{c}\text { VAS - 3rd } \\
\text { follow up }\end{array}$ & $\begin{array}{c}\text { VAS - 4th follow } \\
\text { up }\end{array}$ \\
\hline PRP & 7.2 & 4.7 & 3.7 & 5.53 & 4.57 \\
\hline STEROID & 7.06 & 4.23 & 3.62 & 4.53 & 4.05 \\
\hline $\mathrm{p}$ value & 0.085 & 0.048 & 0.051 & 0.06 & 0.067 \\
\hline
\end{tabular}

In both the groups there is significant difference in the VAS scores $p<0.001$ from the time of presentation to the first follow up in both the groups this further improved at the second follow up significant difference $\mathrm{p}<0.001$ after which it remained a constant, between the two groups. There is no difference with a chi-squares $p$ value is less than 0.05 at all times.

Table 4: FADI at various intervals

\begin{tabular}{|l|c|c|c|c|}
\hline & $\begin{array}{c}\text { FADI -At } \\
\text { presentation }\end{array}$ & $\begin{array}{c}\text { FADI - 1st } \\
\text { follow up }\end{array}$ & $\begin{array}{c}\text { FADI -3rd } \\
\text { follow up }\end{array}$ & $\begin{array}{c}\text { FADI - 4th follow } \\
\text { up }\end{array}$ \\
\hline PRP & 36.33 & 76.33 & 80 & 71.2 \\
\hline Steroid & 36.8 & 79.38 & 84.68 & 79.39 \\
\hline$p$ value & 0.901 & 0.082 & 0.0751 & 0.052 \\
\hline
\end{tabular}

In both the groups there is significant difference $\mathrm{p}<0.001$ from the time of presentation to the first follow up in both the groups FADI score. 


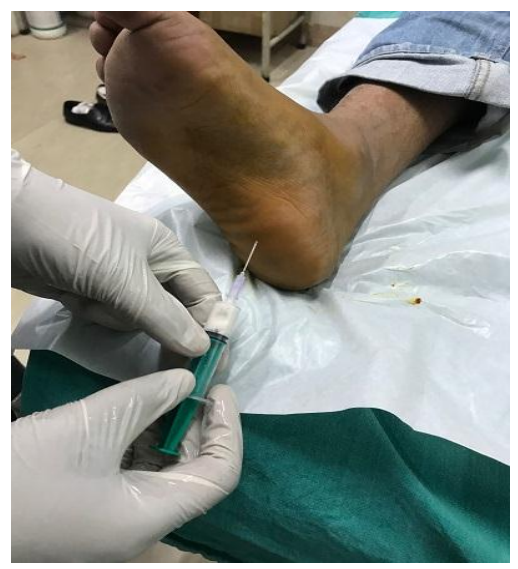

Fig. 1:

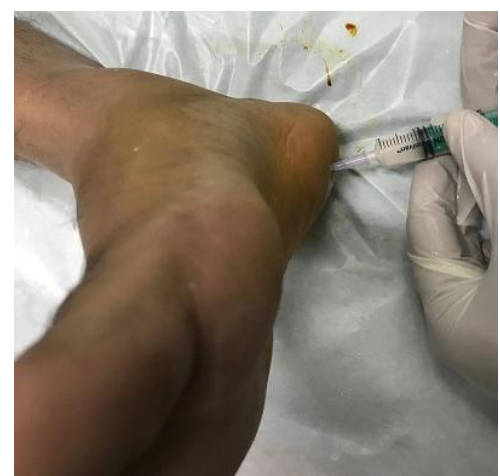

Fig. 2:

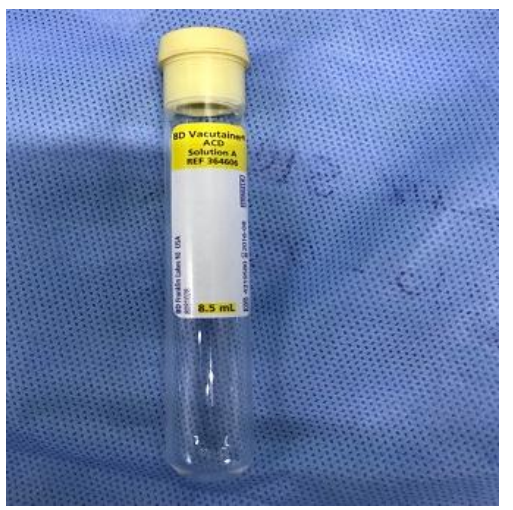

Fig. 3:

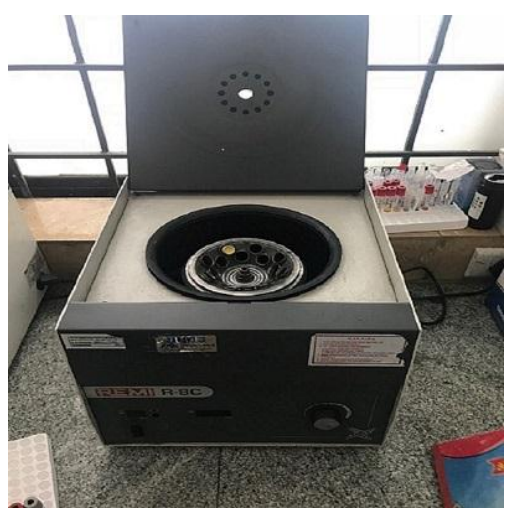

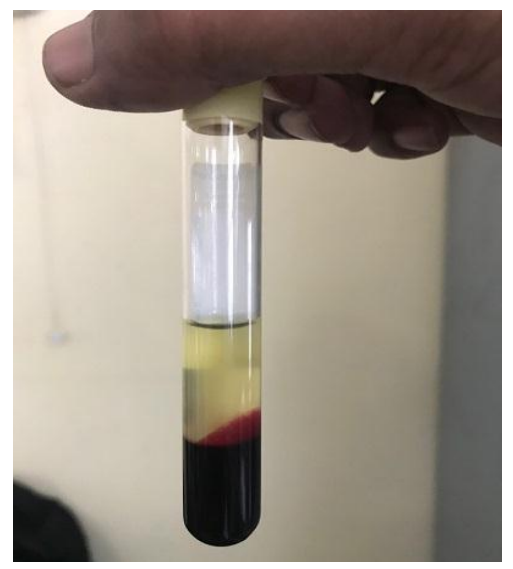

Fig. 5:

\section{Discussion}

In simple terms tendinopathy refers to a condition that occurs within a tendon as a result of repeated trauma and overuse, the condition is painful and most often causes affects the quality of life in the working population thus affecting both physically and socially. Among all the known tendinopathies plantar fasciitis and tennis elbow account for most cases. ${ }^{25}$

In the present day corticosteroid injections are considered as the treatment of choice for tendinopathies as they decrease the inflammatory process thus signs rubor, dolor and color, but they are known for complications like tendon damage and can even cause tendons to rupture. One of the emerging therapeutic modalities is the use of platelet rich plasma for tendinopathies having the advantage of being autologous and concentrated that are rich in growth factor proteins that promote the healing process.

The study is done to find the efficacy of these two modalities at the Department of Orthopaedics Rajarajeshwari Medical College And Hospital, Bangalore on those cases with chronic plantar fasciitis who have satisfied the inclusion criteria and exclusion criteria. Below we have compared our findings with other findings.

\section{Summary}

The study was done to find the efficacy of these two modalities at the Department of Orthopaedics Rajarajeshwari Medical College and Hospital, Bangalore on those cases with chronic plantar fasciitis who has satisfied the inclusion criteria and exclusion criteria. Below we have compared our findings with other findings.

The least age in our study is 18 years and the maximum age is 55 years, the mean in the steroid group is 37. 3 and in the PRP group is 37.43 , the most common group is $31-40$ years $(53 \%)$, right side was most commonly involved $52 \%$. The most common occupation involved was farmers $32 \%$ followed by shop keepers $22 \%$ then teachers $17 \%$ all had standing

Fig. 4: 
for long hours, overall $56 \%$ were overweight, the rest $17 \%$ have BMI of 22.

In both the groups there is significant difference in the VAS scores $p<0.001$ from the time of presentation to the first follow up in both the groups this further improved at the second follow up, significant difference $\mathrm{p}<0.001$ after which it remained a constant, between the two groups there is no difference with a chi-squares $p$ value less than 0.05 at all times.

In both the groups there is significant difference $\mathrm{p}<0.001$ from the time of presentation to the first follow up in both the groups FADI score.

\section{Conclusion}

Plantar fasciitis has a predilection to those who have use of lower limb for a long time in the form of standing or walking.

The pain is the most common compliant and this is effectively controlled with a single dose of steroid or platelet-rich plasma.

PRP has a better effect on pain in patients with longer duration as compared to local steroids but the difference is not statistically significant.

The making of PRP is cumbersome but the patients did prefer it to steroids due to the adverse effects.

\section{References}

1. Taunton J, Ryan M, Clement D, McKenzie D, LloydSmith D, Zumbo B. A retrospective case-control analysis of 2002 running injuries. Br J Sports Med, 2002,36:95101.

2. Buchbinder R. Clinical practice. Plantar fasciitis. N Engl J Med, 2004, 350:2159-2166.

3. Lemont H, Ammirati KM, Usen N. Plantar fasciitis: a degenerative process (fasciosis) without inflammation. $J$ Am Podiatr Med Assoc. 2003;93(3):234-237.

4. Mark W. Scioli, MD. Platelet-rich Plasma Injection for Proximal Plantar Fasciitis. Tech Foot \& Ankle 2011;10:710).

5. Gollwitzer H, Diehl P, von Korff A et al (2007) Extracorporeal shock wave therapy for chronic painful heel syndrome: a prospective, double blind, randomized trial assessing the e \& cacy of a new electromagnetic shock wave device. J Foot Ankle Surg 46:348-357.

6. Malay DS, Pressman MM, Assili A et al (2006) Extracorporeal shockwave therapy versus placebo for the treatment of chronic proximal plantar fasciitis: results of a randomized, placebo-controlled, double-blinded, multicenter intervention trial. J Foot Ankle Surg 45:196210.

7. Speed CA (2004) Extracorporeal shock-wave therapy in the management of chronic soft-tissue conditions. J Bone Joint Surg 86B:165-171.

8. Crawford F, Thomson C: Interventions for treating plantar heel pain (Review). Cochrane 2003.

9. Kalaci A, Cakici H, Hapa O, et al. Treatment of plantar fasciitis using four different local injection modalities: a randomized prospective clinical trial. J Am Podiatr Med Assoc. 2009;99(2):108-113.

10. Eppley BL, Woodell JE, Higgins J (2004) Platelet quantification and growth factor analysis from platelet rich plasma: Implications for wound healing. Plast Reconstr Surg 114:1502-1508.
11. Filardo G, Kon E, Della Villa S, Vincentelli F, Fornasari PM, Marcacci M (2010) Use of platelet-rich plasma for the treatment of refractory jumper's knee. Int Orthop 34:909-915.

12. Foster TE, Puskas BL, Mandelbaum BR et al (2009) Platelet-rich plasma: from basic science to clinical applications. Am J Sports Med 37:2259-2272.

13. Gollwitzer H, Diehl P, von Korff A et al (2007) Extracorporeal shock wave therapy for chronic painful heel syndrome: a prospective, double blind, randomized trial assessing the e \& cacy of a new electromagnetic shock wave device. J Foot Ankle Surg 46:348-357.

14. Gosens T, Den Oudsten BL, Fievez E, van't Spijker P, Fievez A (2012) Pain and activity levels before and after platelet-rich plasma injection treatment of patellar tendinopathy: a prospective cohort study and the influence of previous treatments. Int Orthop 36:19411946.

15. Hall MP, Band PA, Meislin RJ et al (2009) P latelet-rich plasma: current concepts and application in sports medicine. J Am Acad Orthop Surg 17:602-608

16. Mishra A, Pavelko T (2006) Treatment of chronic elbow tendinosis with buffered platelet-rich plasma. Am J Sports Med 34:1774-1778.

17. Ferrari M, Zia S, Valbonesi M. A new technique for hemodilution, preparation of autologous platelet intraoperative blood salvage in cardiac surgery. Int J Artif Organs 1987;10:47-50.

18. Hall MP, Brand PA, Meislin RJ, et al. Platelet-rich plasma: Current concepts and application in sports medicine. J Am Acad Orthop Surg 2009;17:602-609.

19. Marx R, Garg A. Dental and craniofacial applications of platelet-rich plasma. Carol Stream: Quintessence Publishing Co, Inc;2005.

20. Mishra A, Pavelko T. Treatment of chronic elbow tendinosis with buffered platelet-rich plasma. Am J Sports Med 2006;10(10):1-5.

21. Barrett S, Erredge S. Growth factors for chronic plantar fascitis. Podiatry Today 2004;17:37-42.

22. Marx RE. Platelet-rich plasma: evidence to support its use. J Oral Maxillofac Surg 2004;62:489-496.

23. Martinelli N, Marinozzi A, Carnì S, Trovato U, Bianchi A, Denaro V. Platelet-rich plasma injections for chronic plantar fasciitis.

24. Hicks JH, The mechanics of the foot II: the plantar aponeurosis and the arch, Journal of Anatomy. 1954, 88:25-30.

25. Huffer D, Hing W, Newton R, Clair M. Strength training for plant ar fasciitis and the intrinsic foot musculature: A systematic review. Physical Therapy in Sport. 2017 Mar 31;24:44-52. 